\title{
Prédiction juvénile de la qualité du bois de hêtre
}

\author{
G. NEPVEU \\ I.N.R.A., Station de Recherches sur la Qualité des Bois \\ Centre de Recherches forestières de Nancy, \\ Champenoux, F 54280 Seichamps
}

\begin{abstract}
Résumé
L'article présente des corrélations phénotypiques juvénile-adulte calculćes sur 26 hêtres d'un peuplement naturel vieux de 100 ans environ.

Les caractéristiques étudiées sont : les retraits axial et tangentiel, la densité, l'homogénéité du bois et la vitesse de croissance.

Une sélection précoce n’apparaît possible que pour la densité du bois. étudiées.

Des liaisons inter et intra-arbre entre les différentes caractéristiques mesurées sont.
\end{abstract}

\section{1. - Introduction}

On ne trouve pas dans la littérature forestière d'études sur le déterminisme précoce de la qualité du bois de hêtre, hormis quelques indications de Koltzenburg (1967 a) et de Bordeaux (1977). C'est une lacune quil nous a semblé important de contribuer à combler.

En effet, la régénération artificielle, maintenant assez utilisćc pour cette essence, nous donne le moyen d’intervenir sur la composition génétique de la population que l'on va installer.

Dans cette perspective, il est souhaitable de pouvoir juger le plus tôt possible la qualité des génotypes, en étant assuré que leur classement ne se modifiera pas notablement jusqu'à l'âge d'exploitabilité. C'est le but de l'étude des liaisons juvénileadulte que nous présentons maintenant.

Faute d’un matériel adéquat qui nous permette de tenir compte dans lensemble de la variabilité des effets génétiques, nous parlerons de corrélations phénotypiques, et non génétiques. En toute rigueur, la réponse à la question posée : "le jugement précoce de la qualité du bois de génotypes est-il possible?» devrait être apportée en étudiant la similitude des cíassements, aux états juvénile et adulte, de moyennes de descendances ou de clones (corrélations génétiques ou génotypiques), et non d'individus (corrélations phénotypiques). 
Malgré ces réserves, il faut toutefois indiquer au lecteur qu'en matière de liaisons juvénile-adulte pour la qualité du bois, il y a rarement une grande différence entre les deux types de coefficients (NEPVEU, 1976 ; NEPVEL \& Birot, 1979 ; Birot \& NePVeU, 1979), ce qui n'est pas le cas, loin s'en faut, des corrélations mettant en jeu des caractères différents.

Les caractéristiques que nous avons étudiées interviennent à des degrés divers dans la définition de la qualité du produit :

\section{Densité du hois}

Les hêtres de faible densité ont un plan ligneux riche en vaisseaux qui confère au matériau une tendreté recherchée surtout pour le déroulage, l'ébénisterie et le travail du bois en sculpture.

\section{Homogénéité du bois}

Elle intervient dans la qualité des états de surface des produits finis, les feuilles de déroulage tout particulièrement. Cependant, pour le hêtre, dont le bois est assez homogène, cette caractéristique s'avère assez secondaire par rapport à la densité.

\section{Retrait du bois}

Il quantifie son instabilité dimensionnelle, défaut cardinal du bois.

Nous avons mis également en avant dans ce travail la largeur des cernes, caractère dont le poids ne doit pas être négligé, même si parfois, pour certaines essences, il s'avère opposé à un objectif « qualité ».

\section{2. - Matériel et méthodes}

Vingt-six hêtres de la forêt domaniale de Lyons, près de Rouen, ont été utilisés dans ce travail.

Leur âge avoisine 100 ans; ils proviennent d'une même parcelle de 28 ha (parcelle IB1) régénérée naturellement et maintenue assez dense (6 éclaircies en 100 ans).

Ils ont été échantillonnés au hasard parmi 150 chablis : on peut penser que cela n'a pu biaiser la représentation de la parcelle que si ces chablis correspondent à des sujets ayant une architecture ou des dimensions particulières (des études complémentaires à ce sujet seraient utiles). Les circonférences à $1.30 \mathrm{~m}$ s'échelonnaient entre 124 et $231 \mathrm{~cm}$ (moyenne : $176 \mathrm{~cm}$ ), les hauteurs entre 32 et $40 \mathrm{~m}$ (moyenne : $35,7 \mathrm{~m})$.

L'abattage a lieu en janvier 1973. 
Sur chaque tige, on a prélevé 2 rondelles, l'une à $1,30 \mathrm{~m}$, l'autre à $40 \mathrm{~cm}$ de hauteur, de façon à éviter lempattement, tout en ayant le bois formé le plus tôt possible dans la vie de larbre.

Sur chaque rondelle, suivant un rayon orienté au nord, deux barrettes parallélépipédiques jumelles ont été prélevées à laide de scies-fraises jumelées (THIERCEI.IN F. \& Perrin J.R., 1972), lune de $5 \mathrm{~mm} \times 5 \mathrm{~mm}$, lautre de $1 \mathrm{~cm} \times 2 \mathrm{~cm}$. Lorientation a été choisie de façon à éviter un éventuel tois de tension sur la face ouest des troncs, dans cette région où, à cause des vents d'ouest dominants, une anisotropie est-ouest est possible.

Les mesures ont porté, pour les deux types d'éprouvettes, sur les 20 cernes les plus proches du cœur (bois juvénile) et les 20 cernes les plus proches de l'écorce (bois adulte).

Les mesures suivantes ont été effectuées:

\section{Barrettes de $1 \mathrm{~cm} \times 2 \mathrm{~cm}$}

Découpe cerne par cerne au ciseau à bois, puis mesure de liinfradensité (poids anhydre rapporté au volume saturé) par la méthode de saturation intégrale (KEYLWERTH R., 1954). Lorsque la séparation de cernes très fins d'âge $n$ et $n+1$ n'était pas possible, l'infradensité a été déterminée sur l'ensemble et affectée aux deux cernes.

\section{Barrettes de $5 \mathrm{~mm} \times 5 \mathrm{~mm}$}

Mesure au capteur des retraits axial et tangentiel (retraits entre l'état saturé et 12 p. 100 d'humidité). En appelant $\mathrm{N}$ l'âge des arbres, les zones considérées ont été, côté écorce, celles correspondant aux âges $\mathrm{N}-19$ à $\mathrm{N}$, puis $\mathrm{N}-19$ à $\mathrm{N}-10$, $\mathrm{N}-9$ à $\mathrm{N}-5, \mathrm{~N}-4$ à $\mathrm{N}$, et côté cœur, les zones correspondant aux fourchettes d’âge suivantes : 1 à 20 ans, puis 1 à 5 ans, 6 à 10 ans, 11 à 20 ans (pour la méthodologic, voir Polge H. et al., 1973).

Toutes ces barrettes (côté coeur et côté écorce) ont été découpées en plaquettes de $2 \mathrm{~mm}$ d'épaisseur aux fins de mesure de la densité cerne par cerne du bois par microdensitométrie (Тнову M., 1975). Une finesse de l'échantillon de cet ordre était nécessaire afin d'améliorer la netteté des radiographies. Les largeurs de cernes et les contrastes de densité (différence entre densités maximum et minimum de cerne) ont été relevés dans le même temps.

Enfin, les plaquettes ont été découpées, côté cour et côté écorce, en trois zones homologues de celles pour lesquelles les retraits ont été notés; l'infradensité de ces morceaux a été mesurée. La figure n" 1 résume, pour plus de clarté, lensemble des općrations effectuées. 


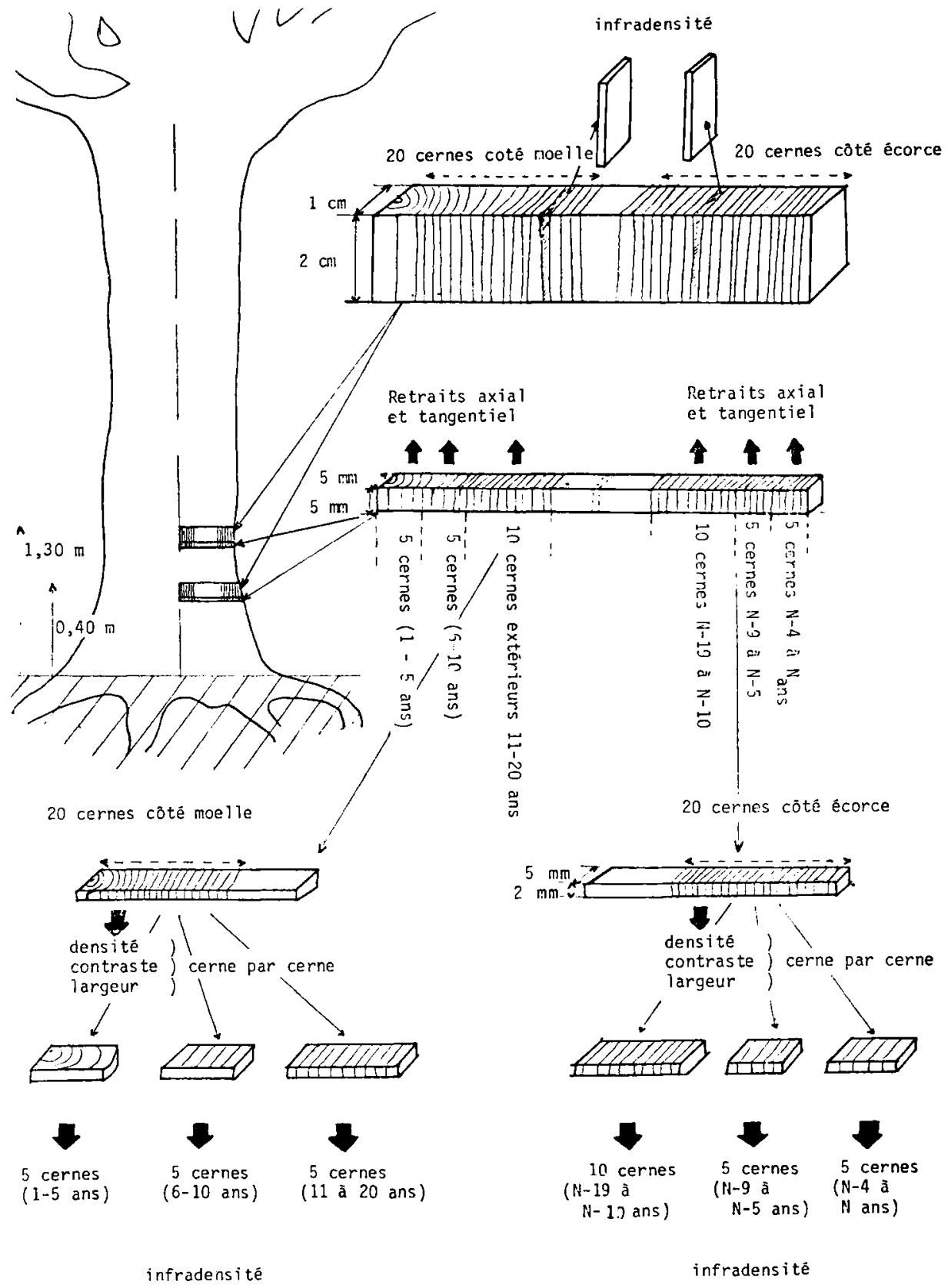

FIG. 1

Echantillonnage à l'intérieur des arbres

Sampling within trees

cernes côté moelle = rings near pith

cernes côté écorce $=$ rings near bark

retrait axial $=$ longitudinal shrinkage

retrait tangentiel $=$ tangential shrinkage

infradensité $=$ basic density

densité $=$ density

contraste $=$ density contrast

largeur $=$ ring width 


\section{Remarques}

- l'infradensité du bois d'une barrette comportant $n$ cernes dont on connaît individuellement les infradensités (donc les poids anhydre et saturé $P_{o_{i}}$ et $P_{s_{i}}$ ) a été calculée comme suit :

$$
d_{n}=f\left(\sum_{i=1}^{n} P o_{i}, \sum_{i=1}^{n} P_{s_{i}}\right)
$$

l'expression $\mathrm{f}(\mathrm{x}, \mathrm{y})$ étant la formule de Keylwerth qui s'écrit en l'occurrence :

$$
\mathrm{d}_{11}=\frac{1}{\frac{\sum_{\mathrm{i}=1}^{\mathrm{n}} \mathrm{P}_{\mathrm{S}_{\mathrm{i}}}}{\sum_{\mathrm{i}=1}^{\mathrm{n}} \mathrm{Po}_{\mathrm{i}}}-0,347}
$$

- la densité (obtenue al densitomètre) d'une barrette comportant n cernes dont on connaît individuellement les densités $d_{i}$ et les largeurs $l_{i}$ a été calculéc comme suit :

$$
d_{11}=\frac{\sum_{i=1}^{n} d_{i} l_{i}}{\sum_{i=1}^{n} l_{i}}
$$

- le contraste de densité et la largeur de cerne sur une barrette comportant n cernes dont on connait individuellement les contrastes $c_{i}$ et les largeurs $l_{i}$ ont été calculés comme suit :

$$
c_{n}=\frac{\sum_{i=1}^{n} c_{i}}{n} \text { et } \quad l_{n}=\frac{\sum_{i=1}^{n} l_{i}}{n}
$$

\section{3. - Résultats et commentaires}

\section{1. - Effet de l'âge sur les caractéristiques étudiées}

3.11. Densité $d u$ bois (infradensité et densité obtenue au microdensitomètre)

Le tableau 1 permet de comparer les densités du bois de plusieurs zones à l'intérieur de l'arbre:

- comparaison globale des 20 cernes côté écorce aux 20 cernes côté cœur; 


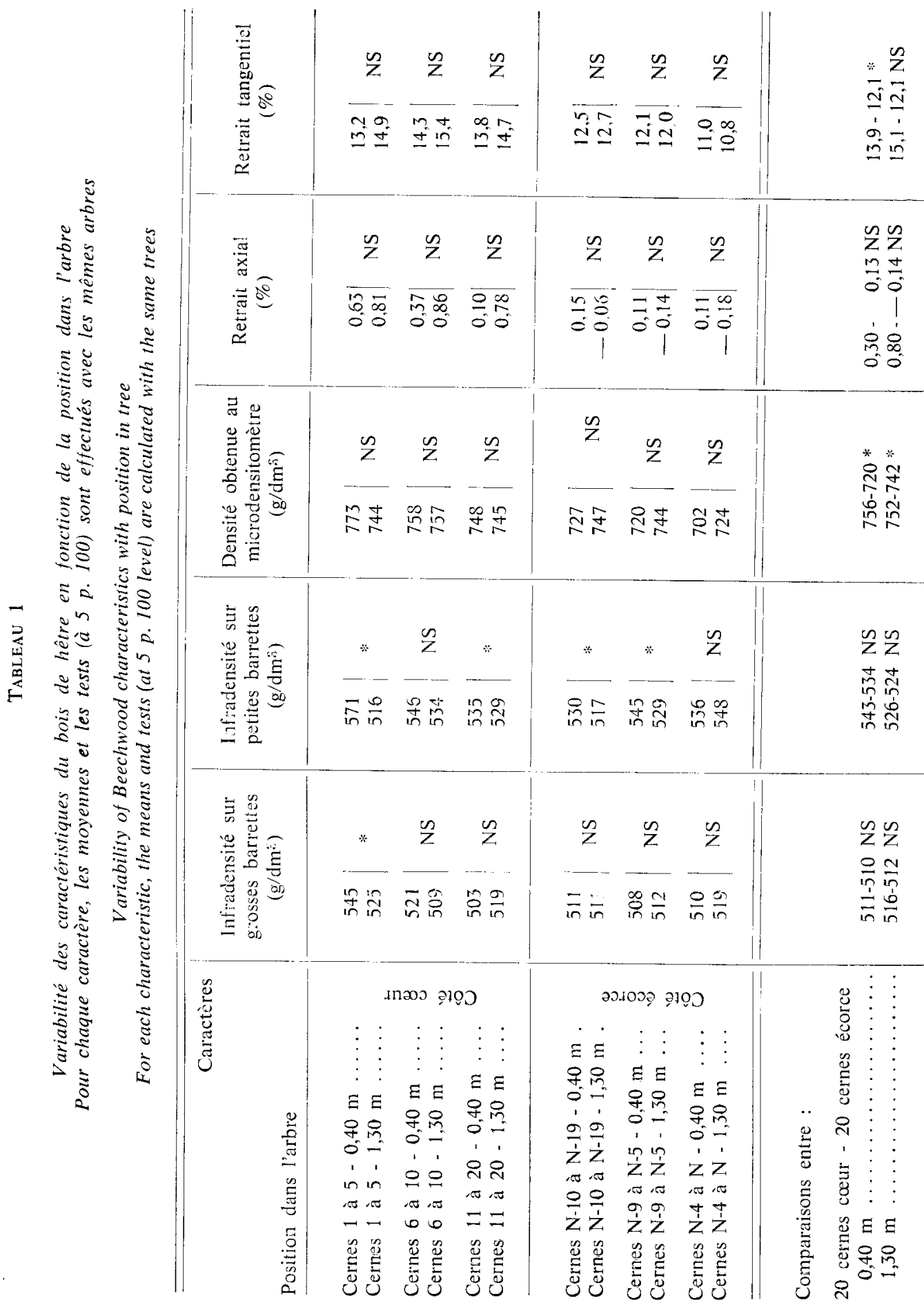



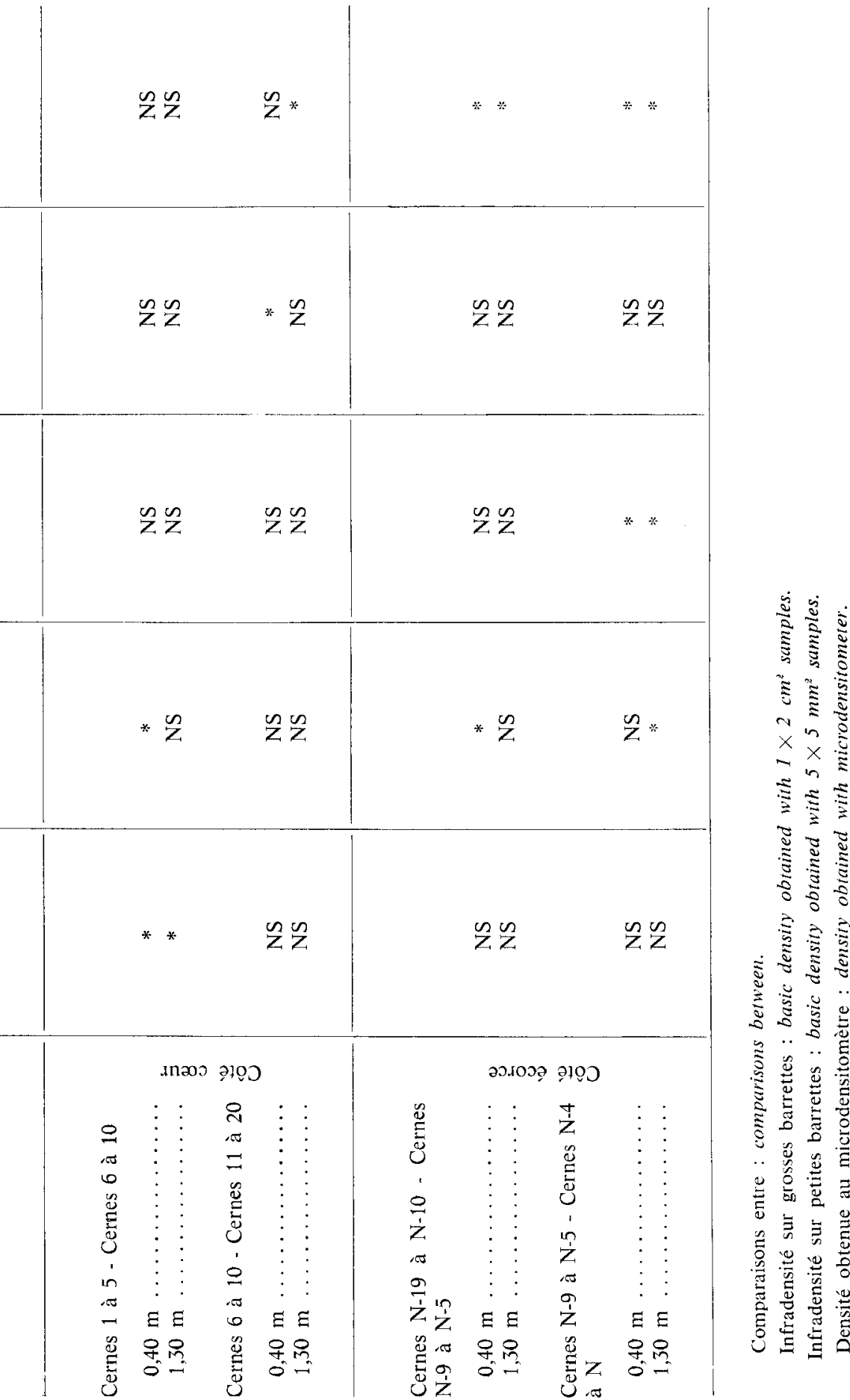

ćcorce ;

- comparaison entre elles des zones de 5 ou 10 cernes côté caur ou côté

- comparaison des zones homologues sur le plan de l'âge par rapport à lassise génératrice (côté écorce) et par rapport à la moelle (côté cœur) et situées à deux hauteurs différentes dans l'arbre.

Les tests effectués sont des comparaisons de moyennes appariées (" quand les moyennes diffèrent au seuil de 5 p. 100, NS dans le cas contraire).

Le tableau 1 fait apparaître des résultats différents suivant les méthodes de mesure employées. Ceci peut s'expliquer en premier lieu par le fait que la densité mesurée au densitomètre est une densité du bois sec à l'air (densité à 12 p. 100 d'humidité/volume à 12 p. 100 d'humidité), alors que les infradensités sont des rapports de poids de bois anhydre au volume saturé; ces deux grandeurs intègrent donc le retrait du bois et son humidité de façon différente; il faut rappeler en outre que l'infradensité obtenue par la pesée du même échantillon à l'état anhydre et à l'état saturé en appliquant la formule de KEYLwERTH néglige les substances solubles. présentes dans le bois jusqu'à concurrence de 4 p. 100 de son poids et qui, lors de la saturation par ébullition dans l'eau, se trouvent dissoutes dans celle-ci. Les répétabilités différentes des types de mesures interviennent sans doute également (un article méthodologique sera rédigé à ce propos). Il ne s’agit pas en tout cas d'un effet de l'échantillonnage, les mêmes arbres ayant été considérés, en excluant ceux pour lesquels l'un des caractères faisait défaut (moyennes sur les 12 mêmes arbres).

En ne tenant compte que des deux infradensités (dont les résultats sont d'ailleurs cux-mêmes un peu différents), on conclut globalement qu'aussi bien à $0,40 \mathrm{~m}$ qu'à $1,30 \mathrm{~m}$, les 20 cernes côté cơur et les 20 cernes côté écorce ont sensiblement la même infradensité. Côté cœur, pour les 5 premiers cernes, le bois pris à $0,40 \mathrm{~m}$ apparaît plus lourd qu'à $1,30 \mathrm{~m}\left(545\right.$ contre $525 \mathrm{~g} / \mathrm{dm}^{3}$ pour les grosses barrettes, 571 contre $516 \mathrm{~g} / \mathrm{dm}^{3}$ pour les petites barrettes), l'écart pour les autres zones entre les deux hauteurs n'étant pas net. Toujours à $0,40 \mathrm{~m}$, tout près du couur, linfradensité est maximum.

Les valeurs supérieures de linfradensité sur petites barrettes par rapport à celle mesurée sur grosses barretles, a priori surprenantes, s'expliquent assez simplement. En effet, l’infradensité Po/Vs est calculée par la formule

$\frac{1}{\frac{\text { PM }}{\text { PO }}-0,347}$ (KeylwerTh R., 1954, op. cit.)

donc par la double pesée de léchantillon saturé (PM), puis anhydre (PO). Lors de la première pesée, on ôte par un passage rapide sur un papier buvard l'cau déposée à la surface de l'échantillon; or, au cours de cette opération, une partie de l'eau contenue dans les vaisseaux au voisinage de la surface quitte le bois dans une proportion négligeable lorsqu'il s'agit d'échantillons assez conséquents, mais sur des ćprouvettes de faible épaisseur, l'effet parasite est à proportion plus important. Ceci minore PM, donc majore l'infradensité. 
En ce qui concerne la densité du bois sec à laair notée au densitomètre, on relève des résultats sensiblement différents avec une supériorité du côté coeur sur le côté écorce, tant à $0,40 \mathrm{~m}$ quà $1,30 \mathrm{~m}$. Cela pourrait s'expliquer par le fait que, côté cour, le bois renferme un certain nombre de substances liées at processus de duraminisation (on devrait plutôt employer le terme de vieillissement, le mot \& duraminisation » étant réservé aux bois à ceeur distinct, par sa couleur, de l'aubier). On notera que cette différence cour-aubier napparaît pas avec les infradensités, la méthode de mesure du poids saturé par ébullition prolongée entraînant le départ du bois des substances en question. Còté cœur et côté écorce, quels que soient les groupes de cernes, il napparaît pas de différences entre les deux niveaux. Notons que, côté écorce, la densité du bois semble décroître dans les tout derniers cernes.

Toutes ces observations se retrouvent sur les figures 2 et 3 qui présentent les résultats des mesures cerne par cerne (mesures sur grosses barrettes et densitomètre); la légère différence d'échantillonnage n’induit pas de distorsion avece ce qui a été dit ci-dessus, sinon qưil semblerait que la densité à 12 p. 100 soil plus élevée côté écorce à $1,30 \mathrm{~m}$ quà $0,40 \mathrm{~m}$; ce fait n’apparaissait pas significatif sur la figure 2 . quoiqu une tendance semblait se dessiner dans ee sens.

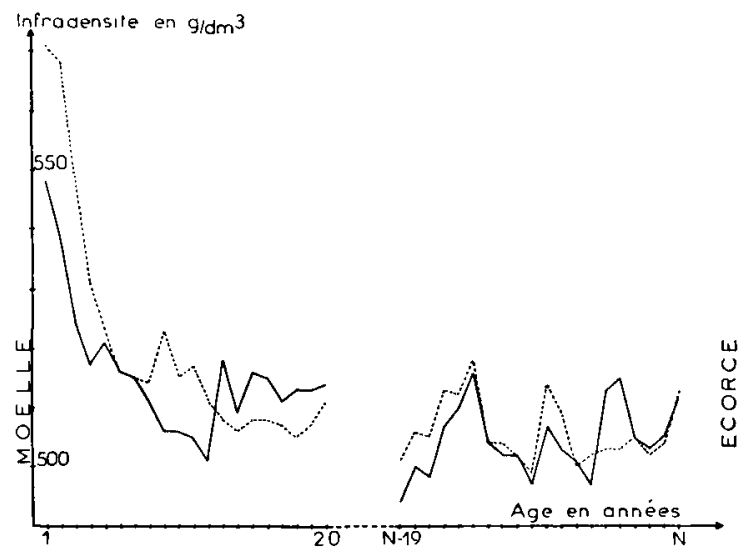

FIG. 2

Evolution de linfradensité sur grosses barrettes avec l'âge; moyenne sur 26 arbres

$V$ ariation of wood basic density obtained with $1 \times 2 \mathrm{~cm}^{2}$ samples in terms of age : means calculated with 26 trees

niveau $1,30 \mathrm{~m}$

niveau $0,40 \mathrm{~m}$

La bibliographie concernant les variations de la densité du bois de hêtre dans l'arbre fournit des résultats et opinions très divers, variant suivant les méthodologies, les échantillonnages utilisés ; cette constatation rejoint bien en cela nos propres observations.

Ainsi, si plusieurs auteurs relèvent une diminution de la densité du cour vers l'écorce (Schwappach A., 1898 ; Nachtigall, 1914 ; Filipovicl J., 1965 ; Trendelenblrg R., 1939 ; Parsa Pajouh D., 1970 sur Fagus orientalis: Cividini R., 1969 ; Tsoumis G., 1958 ; Burger H., 1949-1950; Grossi.er W., 1939 ; Stojanoff V. et 
al., 1958 ; Todorovski S., 1968 sur Fagus moesiaca), d'autres ne retrouvent pas une telle variation (DESCH H.E., 1932; LAURENT P., 1979), ou bien ne lobservent qu'à la partie inférieure du tronc (Göhre K. \& Götze H., 1956).

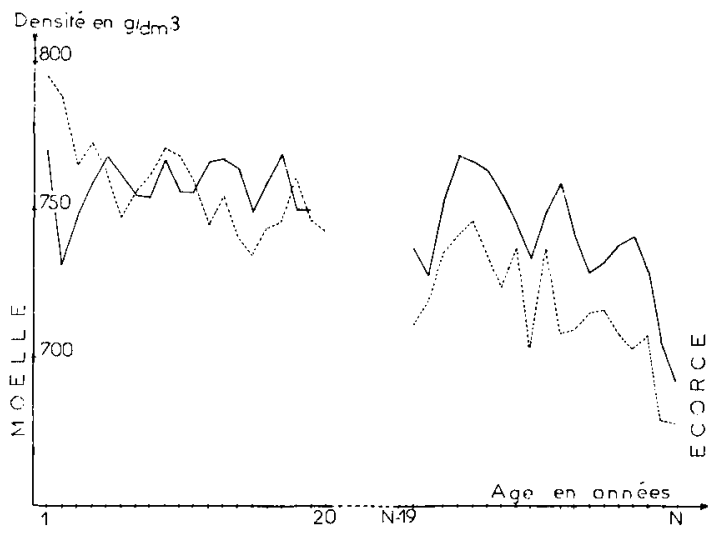

FIG. 3

Evolution de la densité mesurée au microdensitomètre avec l'âge; moyenne sur 13 arbres Variation of density obtained with microdensitomer in terms of age : means calculated with 13 trees.

$\begin{array}{ccc} & \text { niveau } 1,30 \mathrm{~m} \\ \cdots \ldots & \text { niveau } & 0,40 \mathrm{~m}\end{array}$

WoBst H. (1967) relève bien des différences significatives coeur-ćcorce, mais qui jouent tantôt dans un sens, tantôt dans l'autre. Golawski S. (1953), lui, attribue une plus grande densité à la périphérie de l'arbre ou en plein bois (c'est-à-dire le bois situé à peu près à mi-distance entre le centre et la périphérie de l’arbre). Quant à Koltzenburg C. (1967 b), elle observe un accroissement de la densité anhydre de 0 à 40 ans, suivi d'une légère chute pour les hêtres dominés ou codominants, alors que les hêtres dominants semblent peu affectés.

Pour ce qui concerne les variations de densité avec la hauteur dans les tout premiers mètres du tronc, la plupart des études signale une chute sitôt que l'on s'éloigne de la base du fût (Nachtigall, 1914, op. cit.; Grossler W., 1939, op. cit.; Trendelenburg R., 1939, op. cit.; Cividini R., 1969, op. cit.). Le travail de Kol.tzenburg C. (1976 b, op. cit.) indique bien une densité plus importante à $0,30 \mathrm{~m}$ qu'à $0,80 \mathrm{~m}$, mais seulement dans les 25 premiers cernes à partir de la moelle, après quoi l'écart s'estompe.

\subsection{Contraste de densité (figure 4)}

Il augmente régulièrement pendant les 20 premières années, passant de 200 à $250 \mathrm{~g} / \mathrm{dm}^{3}$ tout près du cour, à presque $400 \mathrm{~g} / \mathrm{dm}^{3}$ à 20 ans, avec des valeurs un peu plus élevées à $1,30 \mathrm{~m}$. Les valeurs côté écorce, à peu près constantes et peu différentes entre $0,40 \mathrm{~m}$ et $1,30 \mathrm{~m}$, sont très légèrement supérieures à celles notées à 20 ans côté cœur ; à cet âge donc, le contraste de densité n’a pas encore atteint la valeur qu'il prend dans le bois adulte. 


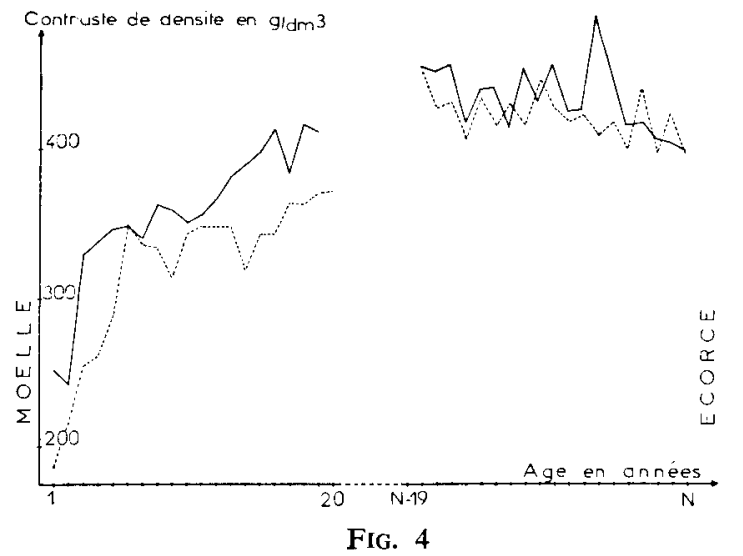

Evolution du contraste de densité avec l'âge: moyenne sur 73 arbres Variation of density contrast in terms of age : means calculated with 13 trees

nivealu $1,30 \mathrm{~m}$ niveau $0,40 \mathrm{~m}$

\subsection{Largeur de cerne (figure 5)}

On notera, en observant la figure 5 , que les largeurs de cernes au voisinage du cœur sont très faibles ( 1 à $2 \mathrm{~mm}$ au cours des 5 premières années), surtout à $0,40 \mathrm{~m}$, ce qui témoigne de la croissance médiocre des jeunes semis de régénération naturelle au début de leur existence.

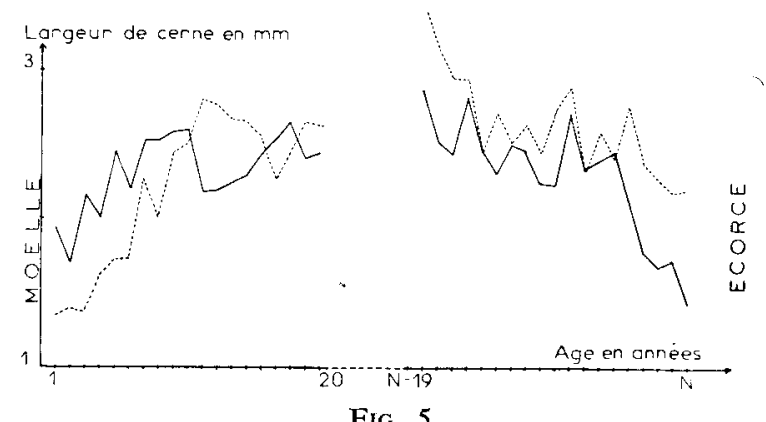

Fig. 5

Evolution de la largeur de cernes avec l'âge : moyenne sur 13 arbres Variation of ring width in terms of age : means calculated with 13 trees niveau $1,30 \mathrm{~m}$ niveau $0,40 \mathrm{~m}$

\subsection{Retraits tangentiel et axial}

Contrairement à ce que pourrait laisser supposer le tableau 1, il n'y a pas de variation du retrait axial du coeur vers l'écorce, ni de bas en haut; on restera cependant réservé sur l'interprétation car la valeur moyenne du caractère étant très faible, 
on peut soupçonner une déficience de la méthodologie que nous avons considérablement améliorée depuis. On peut seulement signaler une diminution de la zone 6-10 ans à la zone 11-20 ans, côté cœur à $0,40 \mathrm{~m}$. Encore faut-il indiquer que ce retrait peut être négatif (c'est-à-dire que le bois gonfle dans le sens axial en passant du point de saturation des fibres à l'état sec à l'air), ce qui fait qu'une diminution peut en fait cacher une augmentation de la proportion d'arbres ayant des retraits négatifs !

En ce qui concerne le bois que nous étudions, Parsa Pajouh D. (1970, op. cit.) relève sur Fagus orientalis un résultat un peu différent du nôtre puisqu'il donne le retrait axial en augmentation lorsqu'on passe du cour vers l'écorce. C'est aussi l'avis de WoBst H. (1967, op. cit.) : tableau 1.

Le retrait tangentiel, quant à lui, süil ne varie pas entre $0,40 \mathrm{~m}$ et $1,30 \mathrm{~m}$ (tableau 1), semble décroître entre le cour et l'écorce tout au moins à $0,40 \mathrm{~m}$ de hauteur. Aux deux niveaux, côté écorce, il décroît même sensiblement à mesure que l'on s'approche de l'assise génératrice. Côté cœur, on ne relève pas de variation entre les différents groupes de cernes si ce n'est, à $1,30 \mathrm{~m}$, entre la zone d'âge 6-10 ans et celle d'âge 11-20 ans, avec une légère diminution.

La bibliographie donne des informations un peu contradictoires pour le retrait dans cette direction lorsque l'on progresse du coeur vers l'écorce, avec tantôt un maintien (WoBsT H., 1967, op. cit.; BUCUR, communication personnelle), ou bien une augmentation (ILIC M., 1974).

\section{2. - Liaisons juvénile-aduite pour les critères de qualité du bois et la vigueur}

Nous avons considéré comme étant du bois adulte la zone de 20 cernes côté ćcorce à $1,30 \mathrm{~m}$ et calculé les corrélations entre ses caractéristiques et celles du bois, côté cœur, soit avec des cernes individuels, soit avec des groupes de 5 ou 10 cernes, afin de déterminer éventuellement lâge à partir duquel on peut prédire, par mesure sur le bois jeune, le classement d'arbres adultes. Les caractéristiques au voisinage du coeur ont été appréciées à $1,30 \mathrm{~m}$, mais également à $0,40 \mathrm{~m}$ pour travailler sur le bois formé le plus tôt possible après la germination de la graine.

\subsection{Caractéristiques mesurées cerne par cerne côté cautur}

(infradensité, densité à l'état sec à l'air, largeur de cerne, contraste de densité)

Des quatre caractères mentionnés (voir figures $6 \mathrm{a}, 6 \mathrm{~b}$ et $7 \mathrm{a}, 7 \mathrm{~b}$ ), seule linfradensité présente une corrélation juvénile-adulte intéressante, sitôt que l’on considère des cernes d'âge supérieur à 10 ans environ à partir de la moelle, pour une hauteur de prélèvement de $1,30 \mathrm{~m}$. Ceci signifie que l'âge à partir de la germination de la graine est sensiblement plus élevé; il faudrait, pour le connaître, ajouter à ces 10 ans, le temps nécessaire au jeune sujet pour atteindre $1,30 \mathrm{~m}$ de hauteur, ce qui, en régénération naturelle, est assez long.

Pour la densité à 12 p. 100 (obtenue au microdensitomètre), les corrélations sont beaucoup moins élevées que celles de la figure 6 a ce qui peut être dû, ainsi que nous le rappelions plus haut, à la nature différente des caractères infradensité et densité à 12 p. 100, mais également à la supériorité des erreurs expérimentales dans le deuxième cas (voir à ce propos l'article méthodologique consacré à cette question). 
bois juvénile pris à $1,30 \mathrm{~m}=$ juvenile wood at $1,30 \mathrm{~m}$ level

bois juvénile prix à $0,40 \mathrm{~m}=$ juvenile wood at $0,40 \mathrm{~m}$ level

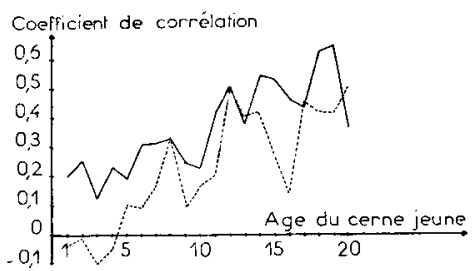

Fig. 6a

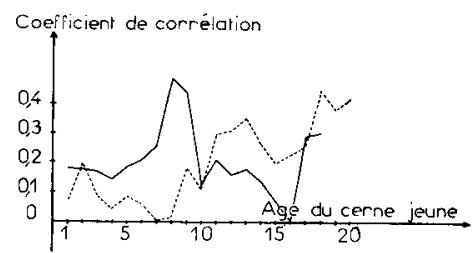

FIG. $6 \mathrm{~b}$

FIG. 6 : Liaisons juvénile-adulte pour la densité du bois appréciée suivant deux méthodes. Bois adulte : 20 cernes côté écorce à $1,30 \mathrm{~m}$. Bois juvénile : cernes d'âges $1,2,3, \ldots$, 20 côté moelle.

Juvenile-mature relationships for wood density obtained with two methods. Mature wood : 20 rings near bark; $1,30 \mathrm{~m}$. Juvenile wood : $1 \mathrm{st}, 2 \mathrm{nd}, \ldots$ 20st ring near pith.

FIG. 6a : Infradensité obtenue sur grosses barrettes - 26 arbres - seuil de signification à 5 p. $100: 0,388$.

Wood basic density obtained with $1 \times 2 \mathrm{~cm}^{3}$ samples - 26 trees - significance level at 5 p. $100: 0,388$.

FIG. 6b : Densité obtenue au microdensitomètre - 24 à 25 arbres - seuil de signification à 5 p. $100: 0,404$ à $0,396$.

Wood density obtained with microdensitometer - 24 or 25 trees - significance level at 5 p. $100: 0,404$ or $0,396$.

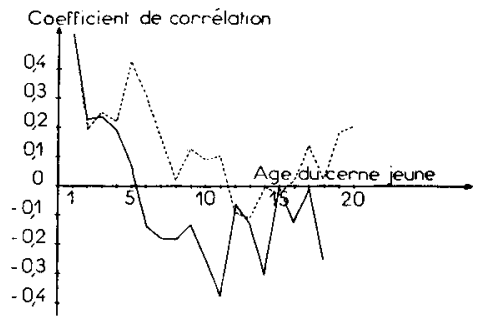

FIG. 7a

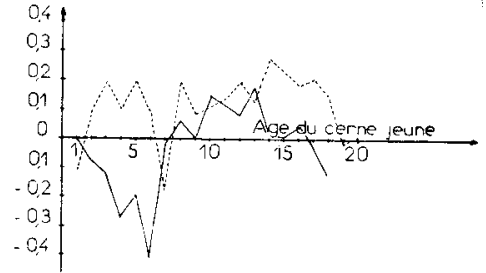

Fig. $7 b$

Fig. 7 : Liaisons juvénile-adulte pour le contraste de densité et la largeur de cerne. Bois adulte : 20 cernes côté écorce à $1,30 \mathrm{~m}$. Bois jévénile : cernes d'âges $1,2,3, \ldots, 20$ côté moelle.

Juvenile-mature relationships for density contrast and ring-width. Mature wood: 20 rings near bark, $1,30 \mathrm{~m}$. Juvenile wood: $1 \mathrm{st}$, 2nd, ... 20st ring near pith.

Fig. 7a : Largeur de cerne - 24 à 25 arbres - seuil de signification à 5 p. $100: 0,404$ à 0,396 .

Ring width - 24 or 25 trees - significance level at 5 p. $100: 0,404$ or 0,396 .

FIG. 7b : Contraste de densité - 24 à 25 arbres - seuil de signification à 5 p. $100: 0,404$ à 0,396 .

Density contrast - 24 or 25 trees - significance level at $5 p .100: 0,404$ or 0,396 . 


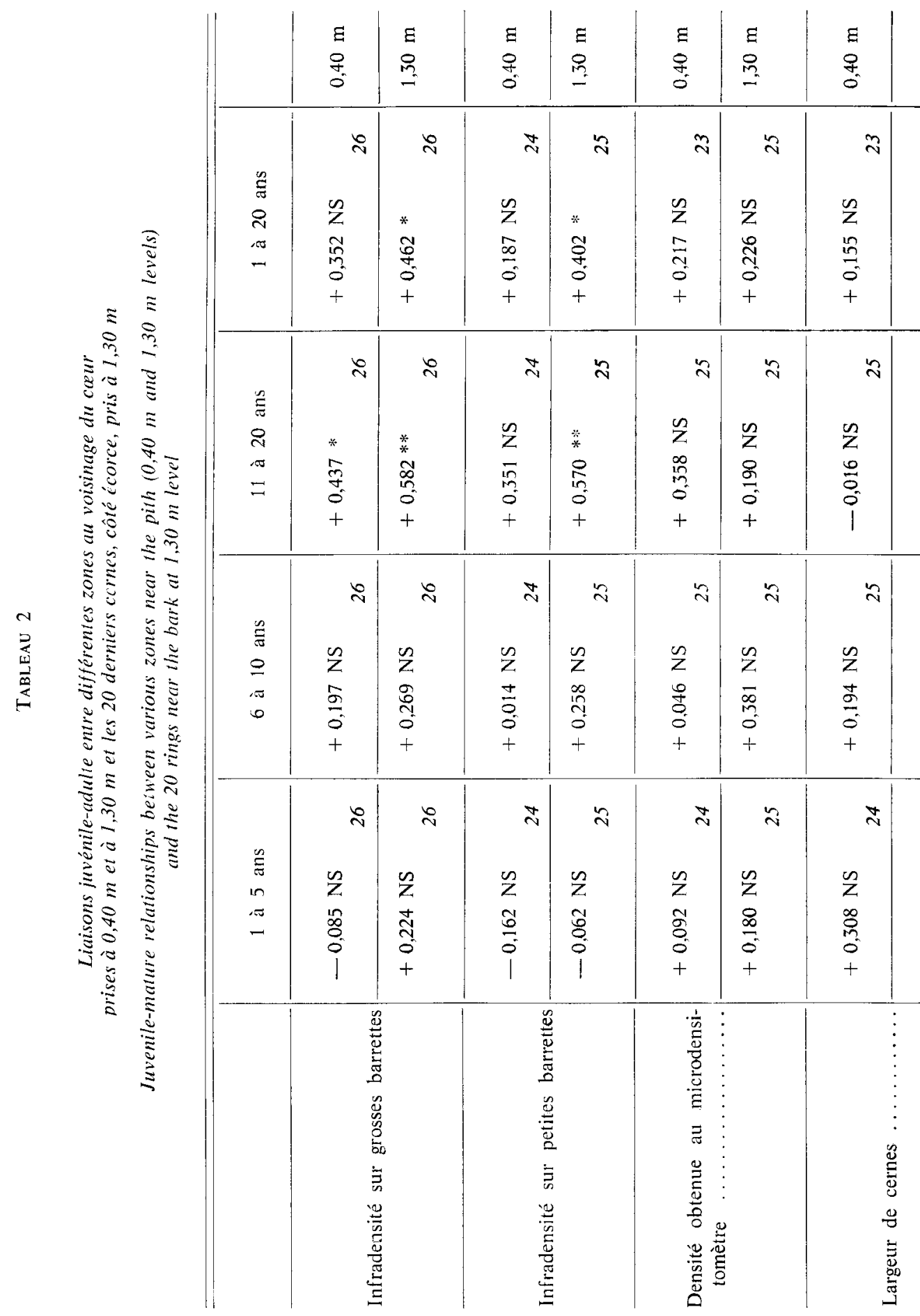




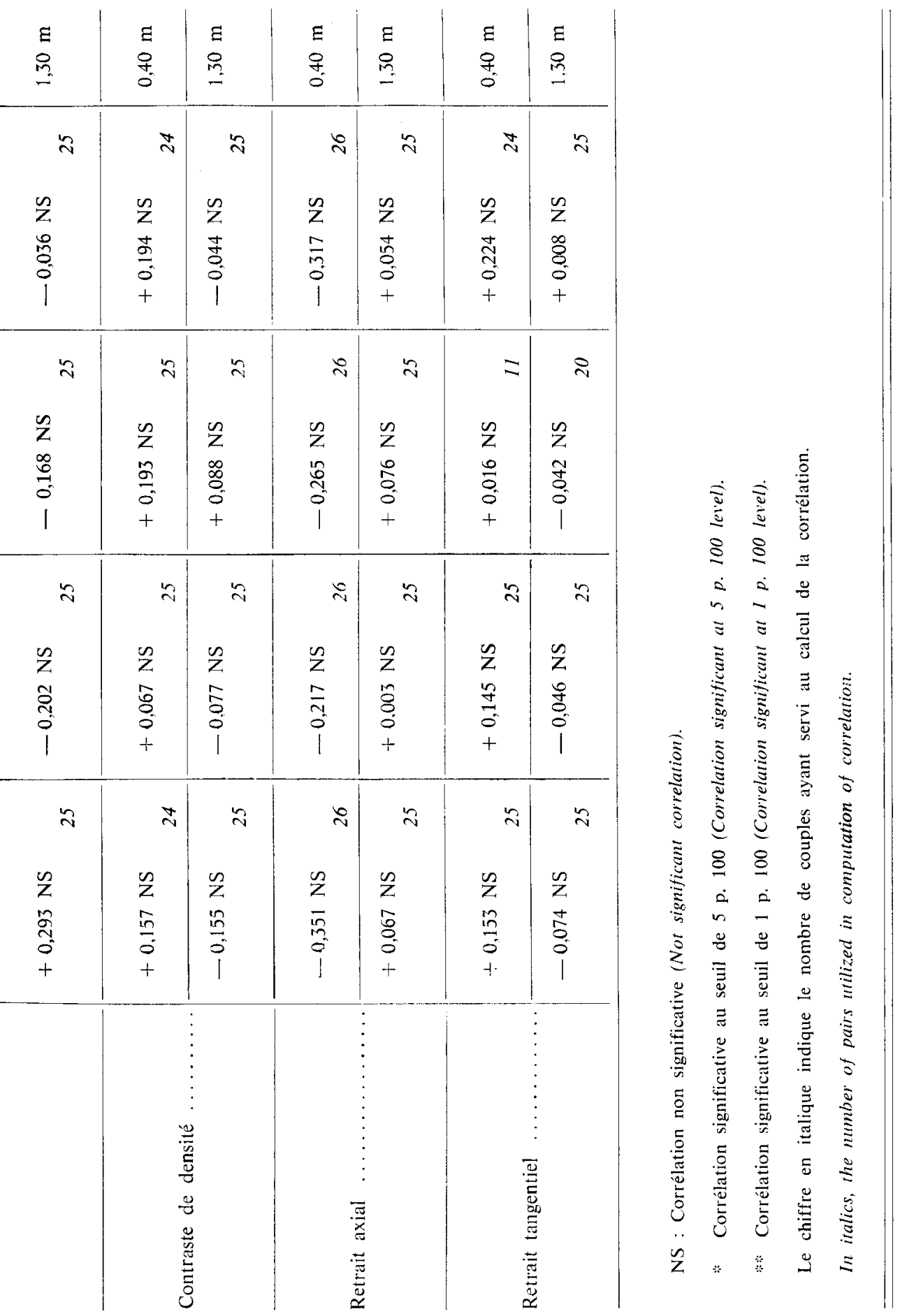


L'observation d'une corrélation juvénile-adulte significative pour l'infradensité mesurée, rappelons-le, à partir d'un seul cerne à proximité du cour, rejoint les deux seuls résultats connus de nous sur le hêtre : ceux de Bordeaux Ch. (1977, op. cit.) et Koltzenburg C. $(1967 \mathrm{~b}$, op. cit.); cette dernière relève une liaison significative entre les densités anhydres des bois formés de 1 à 10 ans et de 40 à 60 ans.

En ce qui concerne la largeur de cerne et le contraste de densité (figures 7 a et $7 \mathrm{~b}$ ), aucune liaison n'apparaît. Signalons toutefois pour les tout premiers cernes quelques valeurs légèrement positives (tant au niveau $0,40 \mathrm{~m}$ qu'au niveau $1,30 \mathrm{~m}$ ), après quoi les courbes rejoignent l'ordonnée zéro. Il n'est pas impossible que le phénotype s'exprime dans le très jeune âge de la même façon qu'à l'âge adulte, avant que des circonstances extérieures (concurrence par exemple) intervenant les années suivantes ne masquent les similitudes de comportement.

Il serait intéressant de pouvoir confirmer ou infirmer ce point de vue en opérant sur des arbres de plantation pour lesquels les conditions de croissance, plus homogènes d'un arbre à l'autre, sont très différentes. En outre, une expérience sur un matériel installé artificiellement présenterait l'avantage de nous rapprocher des conditions dans lesquelles l'améliorateur procédera à des opérations modernes de sélection (test de descendances, test clonal, ...).

\subsection{Caractéristiques mesurées au caur sur des groupes de cernes (tableau 2)}

On considère toujours le bois des 20 cernes côté écorce à $1,30 \mathrm{~m}$ représentatif du bois adulte.

Le tableau 2 montre que seule l'infradensité du bois présente une bonne corrélation juvénile-adulte, à condition toutefois de prendre le bois juvénile à hauteur d'homme, donc à un âge assez avancé depuis la germination de la graine, et point trop au voisinage immédiat de la moelle. Ceci confirme entièrement les observations faites à propos de la figure $6 \mathrm{a}$.

On notera quavec un seul cerne (figure 6 a) ou 10 cernes (tableau 2), les valeurs atteintes par les corrélations ne diffèrent pas sensiblement, ce qui confirme d'une certaine manière la précision de la mesure de l'infradensité portant sur un échantillon d'assez faible volume et représentant un accroissement annuel unique.

\section{3. - Liaisons intercaractères}

\subsection{Liaisons interarbres suivant différentes zones}

Nous avons calculé toutes les corrélations entre les sept caractères figurant au tableau 2 (tableau 3) et ce pour différentes zones dans l'arbre.

Nous observons en premier lieu de bonnes liaisons entre les différentes méthodes de mesure de densité, quelles que soient les zones, et ce malgré la taille très faible de certains échantillons (masse de bois anhydre parfois inférieure à $0,01 \mathrm{~g}$ pour les échantillons découpés sur les petites barrettes). 


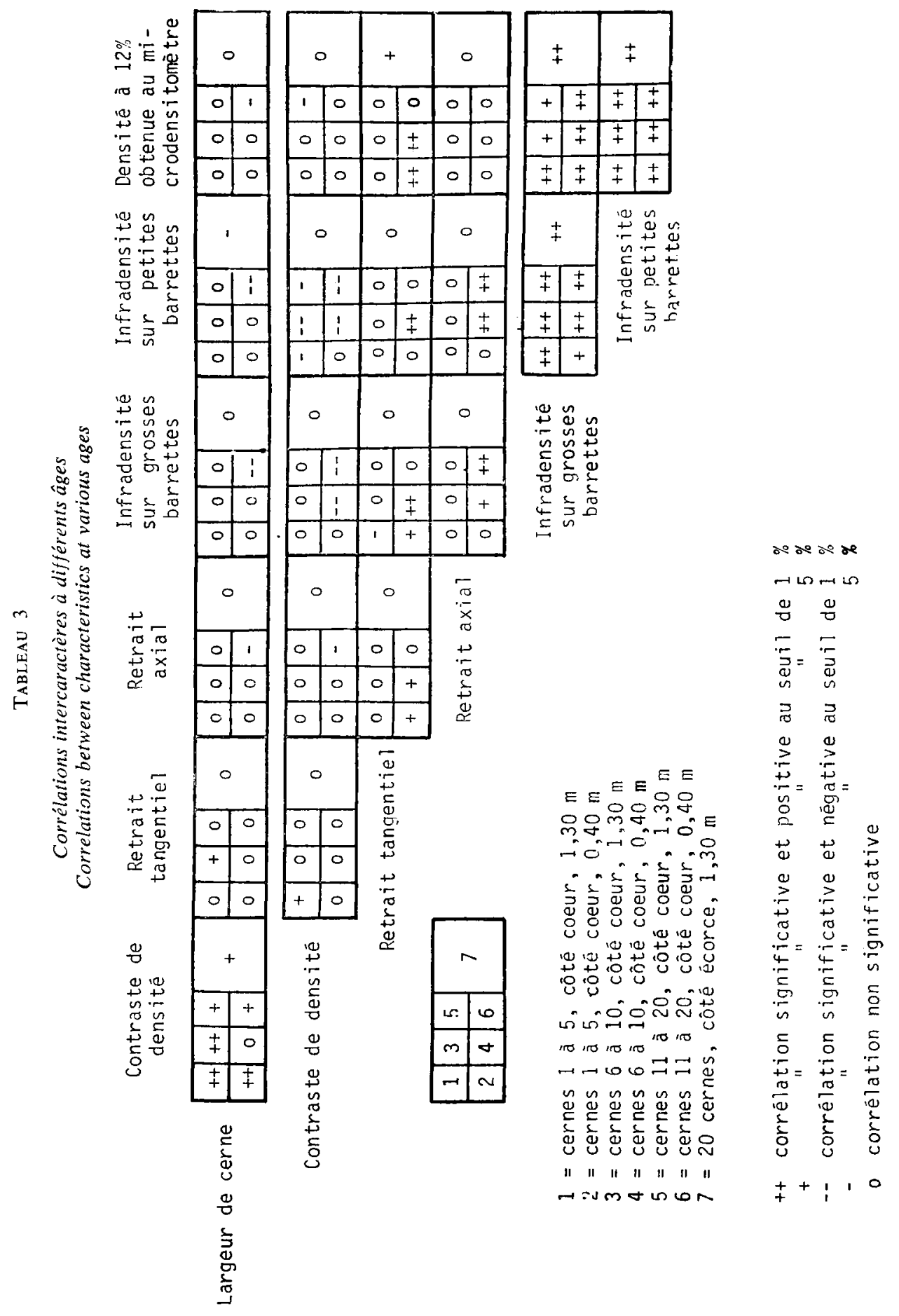


Les corrélations intercaractères concernant les critères de qualité : densité, retrait tangentiel, retrait axial, contraste de densité semblent assez variables suivant la position dans le tronc.

C'est le cas des liens retraits-densité du bois qui ne paraissent s'exprimer qu'à $0,45 \mathrm{~m}$ de hauteur, côté cœur. Ceci modulerait un peu des conclusions assez tranchées qui s'accordent à noter que densité élevée et forts retraits du bois de hêtre vont de pair (Parsa Pajouh D., 1970, op. cit. ; Tsoumis G., 1958, op. cit. ; Venet J., 1972 ; Renaud J.P., 1979 ; Trendelenburg R., 1939, op. cit.; Burger H., 1949-1950, op. cit.; Grossler W., 1939, op. cit.), et se rapprocherait davantage des opinions plus nuancées de Ors Y. (1977), Preney S. (1978) et Trenard Y. \& Gueneau P. (1977).

Le retrait tangentiel n'est lié (positivement) au retrait axial qu'à proximité immédiate de la moelle en bas du fût, alors que deux références (Bordeaux Ch., 1977, op. cit. \& Preney S., 1978, op. cit.) relevaient une telle liaison dans le bois adulte.

Le contraste de densité n'apparaît pas lié avec les deux retraits mesurés. Il semble indépendant des densités côté écorce; côté cour, quelques corrélations négatives apparaissent, ce qui indiquerait que le bois le plus homogène (c'est-à-dire à contraste faible) serait, dans cette zone, le plus dense.

Les hêtres à forte croissance juvénile ou adulte présentent les bois les plus hétérogènes; en revanche, l'impact de la vigueur sur les retraits n'est pas sensible, observation qui rejoint celle de Trenard Y. \& Gueneau (1977, op. cit.).

Les liaisons largeur de cerne-densité ne diffèrent pas sensiblement de zéro, si ce n'est peut-être quelques valeurs négatives à $0,40 \mathrm{~m}$ côté cœur pour la fourchette d'âge 11-20 ans, et à 1,30 m, côté écorce, en prenant en compte l'infradensité mesurée sur petites barrettes. Ces résultats un peu flous rejoignent les informations contradictoires de la littérature concernant le hêtre qui donnent, pour les liaisons interarbres à l'intérieur des stations, des corrélations vigueur-densité tantôt nulles (NACHTIGAll, 1914, op. cit. ; Keller R., Timbal J., Le TACON G., 1976), tantôt positives (Renaud J.P., 1979, op. cit.; Leclerce A., 1977 ; TAYlor F.W., 1969), aussi bien côté cœur que côté écorce (Bordeaux Ch., 1977, op. cit.), tantôt négatives (TsouMis G., 1958, op. cit.; Preney S., 1978, op. cit.).

\subsection{Liaisons intra-arbre pour quelques caractéristiques mesurées au microdensito-} mètre (densité de cerne, contraste de densité, largeur de cerne)

Ces corrélations ont été calculées arbre par arbre sur les 20 cernes du bois adulte $(1,30 \mathrm{~m}$ de hauteur, côté écorce).

Les figures 8 à 10 donnent les diagrammes de fréquence des coefficients de corrélations pour les trois couples de caractères.

\section{Corrélation intra-arbre densité du bois-contraste de densité (figure 8)}

Pour la quasi-totalité des arbres, nous observons une corrélation négative centrée vers - 0,350, alors que la corrélation interarbres était certes négative, mais non 
significative. C'est dire que lorsque l'assise génératrice de l'arbre produit un bois plus dense, le contraste de densité est plus faible, donc le matériau plus homogène.

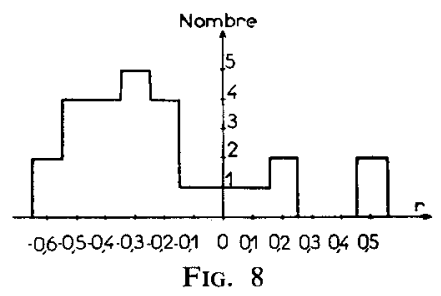

Corrélations intraarbre contraste de densité - densité du bois Diagramme de fréquence des coefficients

La corrélation interarbre valait - 0,299 (non significative) pour 26 couples

Intratree correlations between density contrast and wood density. Histogram of coefficients The intertree correlation value was $-0,299$ (not significant) for 26 pairs

Corrélation intra-arbre contraste de densité-largeur de cerne (figure 9)

Alors que la corrélation interarbres était significative et positive, on observe cette fois une grande majorité de liaisons intra-arbre proches de zéro, avec seulement quelques sujets (5 sur 26) pour lesquels une augmentation de la vitesse de croissance se traduit par une augmentation de l'hétérogénéité du bois.

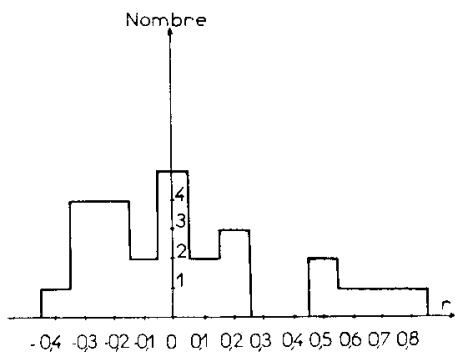

FIG. 9

Corrélations intraarbre contraste de densité - largeur de cerne

Diagramme de fréquence des coefficients

La corrélation interarbre valait $+0,489$ (significative à 5 p. 100) pour 26 couples

Intratree correlations between wood density contrast and ring width. Histogram of coefficients

The intertree correlation value was $+0,489$ (significant at 5 p. 100 level) for 26 pairs

Ceci peut-il s'expliquer par une variabilité de largeur de cernes différente de l'ensemble pour ces quelques arbres? Sans aucun doute, puisque ces individus présentent quelques cernes, soit beaucoup plus étroits que les plus étroits des autres arbres (moins de 5 dixièmes de millimètres), soit sensiblement plus larges que les plus larges des autres arbres (jusqu'à 5 millimètres).

C'est dans ces deux cas extrêmes que le contraste de densité est influencé, soit augmenté pour les accroissements très larges, soit diminué pour les accroissements très fins. En revanche, dans une fourchette de largeur de cernes point trop étendue, cas de la majeure partie de nos échantillons, le contraste de densité n'est pas affecté. 
Corrélations intra-arbre densité-largeur de cerne (figure 10)

Comme dans le cas de la corrélation précédente, on observe, alors que la liaison interarbres n'était pas significative, la séparation de deux populations : l'une, la plus importante, où les arbres présentent une liaison positive largeur de cerne-densité, l'autre - une assez forte minorité — où la liaison est faiblement négative.

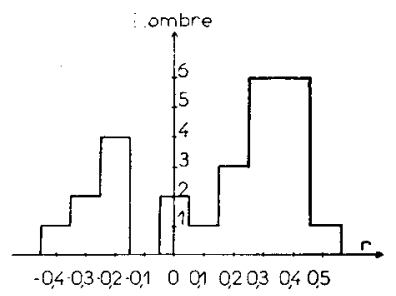

FIG. 10

Corrélations intraarbre densité du bois - largeur de cerne Diagramme de fréquence des coefficients

La corrélation interarbre valait - 0,236 (non significative) pour 26 couples

Intratree correlation between wood density and ring width. Histogram of coefficients The intertree correlation value was - 0,236 (not significant) for 26 pairs

Cependant, contrairement au cas des relations vigueur - contraste de densité, on ne peut affirmer que ce soient les arbres présentant des cas extrêmes sur le plan des largeurs d'accroissements qui voient la densité de ces cernes modifiée. Peut-on en déduire qu'il s'agit de la faculté des génotypes à répondre de diverses façons à des accólérations ou à des diminutions de vigueur? Il serait nécessaire d'engager des recherches plus fines où la source de variation "génétique soit contrôlée pour conclure.

Ces résultats modulent un peu d'assez nombreuses observations indiquant qu'à la suite d'une éclaircie entraînant une augmentation de vigueur, la densité du bois de hêtre s'accroît sensiblement (Cividini R., 1969, op. cit., von PechmanN H., 1958; Grossler W., 1939, op. cit.). Ils ne sont démentis que par Tsoumis G. (1958, op. cit.) qui note une indépendance largeur de cerne - densité, exception faite des cernes très fins (moins de $1 \mathrm{~mm}$ ) pour lesquels la densité fléchirait quand la vigueur augmente.

Nous avons nous-mêmes comparé des groupes de cernes étroits avant éclaircie à d'autres - larges - après éclaircie, et avons noté des augmentations d'infradensité allant jusqu'à 50 , voire $80 \mathrm{~g} / \mathrm{dm}^{3}$, soit 10 à $18 \mathrm{p}$. 100 de plus (résultats non publiés). SchwapPaCH A. (1898, op. cit.) avance des chiffres assez semblables au sujet de la densité anhydre.

Quelques-uns de ces auteurs, von Pechmann notamment, remarquent que cette liaison intra-arbre entre largeur et densité de cerne, globalement vérifiée, varie notablement d'un individu à l'autre, d'une station à l'autre, ce qui rejoindrait bien les observations de la présente étude. 


\section{4. - Conclusion}

Ce travail, qui ne se proposait au départ que d'apprécier les liaisons juvénileadulte pour différents critères de qualité du bois (densité, retrait axial, retrait tangentiel, homogénéité du bois) et pour la vigueur, a connu quelques développements intéressants, en même temps qu'il a soulevé des problèmes méthodologiques qui seront traités dans un prochain article.

- La variation intra-arbre des différents caractères a été étudiée ; elle ne nous apparaît pas très significative, si ce n'est pour le contraste de densité, en regard de la variabilité interarbres.

- Une sélection précoce n'est raisonnable que pour l'infradensité du bois à condition de s'intéresser au bois de plus de 10 ans à partir de la moelle, et en évitant de prendre l'échantillon à une hauteur trop proche du sol. Il faut toutefois rappeler au lecteur que le matériel disponible ne nous permet de parler que de corrélation phénotypique, et non de corrélations génotypique ou génétique, intéressantes pour l'améliorateur. Dans cet ordre d'idées, il est nécessaire de souligner que nous avons travaillé sur un matériel «sauvage», issu de régénération naturelle où les largeurs d'accroissements, surtout juvéniles, sont relativement faibles (2 à $3 \mathrm{~mm}$ ); il n'est pas impossible qu'une étude individualisant des génotypes, entreprise sur des arbres plantés soumis à une concurrence moins forte aurait donné des résultats différents.

- Les liaisons interarbres entre les caractéristiques étudiées, vigueur y compris, apparaissent assez lâches et relativement variables suivant les positions considérées dans l'arbre, à l'exception du couple largeur de cerne - contraste de densité pour lequel une corrélation positive apparaît clairement.

Ces résultats sont intéressants pour le forestier qui n'a pas lieu de redouter pour le hêtre, à la différence du chêne pour lequel, de façon générale, une sylviculture prudente est recommandée, un traitement favorisant la croissance. Ces indications sont valables, il faut le souligner au niveau interarbres; il semble en revanche, si l'on observe les liaisons au niveau intra-arbre, que les conséquences d'une variation de la densité du bois ou de la largeur d'un cerne ou d'un groupe de cernes sur d'autres caractéristiques de ce ou ces accroissements, ne soient pas conformes à ce que pourraient laisser attendre les corrélations interarbres; à tout le moins, on trouve fréquemment des individus échappant à des lois générales.

Ceci indique que l'on doit se garder d'affirmations du genre « cernes étroits = densité du bois élevée », et bien indiquer les sources de variation que l'on prend en considération (peuplements, arbres dans peuplements, accroissements dans arbre). Le cas du chêne rouvre ou pédonculé, pour lequel on a trouvé des individus (rares) alliant bonne vigueur et bonne qualité du bois, malgré une liaison statistique vigueurqualité défavorable, généralement observée chez nos chênes indigènes, est un autre exemple qui incite à cette précaution.

Reçu pour publication en décembre 1980. 


\section{Summary \\ Early prediction of beechwood quality}

This paper presents phenotypic juvenile-mature correlations calculated in twenty-six 100-year-old Beech of a natural high forest.

The characteristics studied are : longitudinal and tangential shrinkage, wood density, homogeneity of wood and growth rate.

An early selection seems possible only for wood density.

Inter- and intratree relationships between the characteristics cited are studied.

\section{Références bibliographiques}

Birot Y., Nepveu G., 1979. Variabilité clonale et liaisons ortets-ramets dans une population d'épicéa. Silvae Genet., 28, 2-3, 37-47.

Bordeaux Ch., 1977. Le hêtre de qualité dans le Haut-Couserans. Rapport de $3^{e}$ année E.N.I.T.E.F., O.N.F., Centre de Saint-Girons

Burger H, 1949-1950. Holz, Blattmenge und Zuwachs; X. Mitteilung : die Buche. Mitt. Schweiz. Anst. forstl. Versuchswes., 26, 2, 419-468.

Cividini R., 1969. Studio technologico sul faggio dell' Appennino Toscano. Contributi Scientifico Pratici per una Migliora conescenza ed Utilizzazione del Legno, 12 (22), 9-38.

Desch H.E., 1932. Anatomical variation in the wood of some dicotyledonous trees. New Phytol., 31, 31-118.

Filrpovici J., 1965. Studiul lemnului. Vol. II. Editura dictatica si pedagogica, Bucaresti.

GöHre K., GöTzE H., 1956. Untersuchungen über die Rohwichte des Rotbuchenholzes. Archiv. Forstwes., 5, 9-10, 716-718.

GolawSKI S., 1953. [Specific gravity and static bending strength of beech in the three main zones of the stem cross-section]. Sylwan, 97 (5).

GRössLer W., 1939. Über Raumgewicht und Holzeigenschaften einiger Rotbuchen aus dem Hochgebirge. Dissertation München. Auszug in Holz-als Roh-Werkstoff, 6 (1943), 3, 81-86.

ILIC M., 1974. [Change in dimensions and internal stresses during air drying of Beech dimension stock]. Pregled Zavod na Technologiju Drveta, Sarajevo, 1-2, 1-18.

Keller R., Timbal J., Le Tacon F., 1976. La densité du bois de hêtre dans le Nord-Est de la France. Influence des caractéristiques du milieu et du type de sylviculture. Ann. Sci. for., 33 (1), 1-17.

Keylwerth R., 1954. Ein Beitrag zur qualitativen Zuwachsanalyse. Holz als Roh- und Werkstoff, 12 (3), 77-83.

Koltzenburg C., 1967a. Die Abhängigkeit der Holzeigenschaften der Rotbuche (Fagus silvatica) von Lichtgenuss, soziologischer Stellung und anderen Wuchsbedingungen. Dissertation zur Erlangung des Doktorgrades der Forstlichen Fakultät des Georg-August Universität zu G.öttingen.

Koltzenburg C., 1967b. Der Einfluss von Lichtgenuss, soziologischer Stellung und des Standortes auf Holzeigenschaften der Rotbuche (Fagus silvatica L.). Holz als Rohund Werkstoff, 25. 
Laurent P., 1979. Contribution à l'étude de l'amélioration des propriétés du bois lamellé imprégné de phénoplaste et comprimé à chaud. Mémoire Ingénieur C.N.A.M. en Chimie Industrielle. Conservatoire National des Arts et Métiers, Centre Régional Agréé de Nancy.

LeCLercQ A., 1977. Relations entre la croissance, la structure et la densité du bois de hêtre. Bull. Rech. agron. Gembloux, 12 (4), 321-330.

NaChtigall, 1914. Vergleichende Untersuchungen an Rotbuchenholz. Z. Forst. u. Jagdwes, 46, 419.

NePveu G., 1976. Les corrélations juvénile-adulte en matière d'étude de la qualité du bois. Document à distribution limitée Station de Recherches sur la Qualité des Bois du C.N.R.F., n" 1976/3.

Nepveu G., Biror Y., 1979. Les corrélations phénotypiques juvénile-adulte pour la densité du bois et la vigueur chez l'épicéa. Ann. Sci. for., 36 (2), 125-149.

Ors Y., 1977. Sélection des variables pertinentes qui agissent sur le comportement rhéologique du bois de Fagus silvatica au moyen d'essais mécaniques, physiques et anatomiques. Thèse de Docteur-Ingénieur. Université Pierre et Marie Curie, Paris VI, 20 juin.

Parsa Pajouh D., 1970. Contribution à l'étude de la qualité du bois de Fagus orientalis de 3 stations de la forêt d'Elbourz au moyen d'essais classiques sur éprouvettes normalisées et d'analyses densitométriques de radiographies. Thèse de Docteur-Ingénieur. Faculté des Sciences de l'Université de Nancy, 16 juillet.

Pechmann H. von, 1958. Die Auswirkung der Wuchgeschwindigkeit auf die Holzstruktur und die Holzeigenschaften einiger Baumarten. Schweiz. Z. Forstwes., 109 (11), 615-47.

Polge H., Keller R., Thiercelin F., 1973. Influence de l'élagage des branches vivantes sur la structure des accroissements annuels et sur quelques caractéristiques du bois de douglas et de Grandis. Ann. Sci. for., 30 (2), 127-140.

Preney S., 1978. Contribution à la typologie des hêtraies du premier plateau du Doubs. Relations avec la qualité du bois. Mémoire de 3" année E.N.I.T.E.F., O.N.F. de Besançon, juillet.

Renaud J.P., 1979. Définition et cartographie de stations dans un massif forestier de hêtre et de chêne : production et qualité. Mémoire de $3^{\varepsilon}$ année E.N.I.T.E.F., O.N.F. de Lons-le-Saunier, juillet.

Schwappach A., 1898. Untersuchungen über Raumgewicht und Druckfestigkeit des Holzes wichtiger Waldbaüme. Verlag von Julius Springer, Berlin.

Stojanoff V., Entcheff E., Göhre K., Götze H., 1958. [Wood density variation within the stem and the influence on it of growth region and site]. Archiv. Forstwes., 7 (12), 953-8, 959-74.

TAYLOR F.W., 1969. The effect of ray tissue on the specific gravity of wood. Wood and Fiber, 1 (2), 142-145.

Thiercelin F., Perrin J.R., 1972. Débit d'échantillons d'épaisseur rigoureusement uniforme à l'aide de scies-fraises jumelées. Ann. Sci. for., 30 (2), 295-299.

Тнову M., 1975. Validité de tests précoces d'appréciation des caractères technologiques du bois de douglas. Application à une plantation comparative de 24 provenances de douglas (Peyrat-le-Château) en liaison avec des caractères morphologiques et auxométriques. Mémoire de $3^{\circ}$ année E.N.I.T.E.F. : Station de Recherches sur la Qualité des Bois, C.N.R.F.

Todorovski S., 1968. [Effect of ecological factors on the physical and mechanical properties of Fagus moesiaca wood from the Karaorman mountains]. God. Zb. Zemjod. Shumar. Fak. Univ. Skopje, 21, 39-59.

Trenard Y., Gueneau P., 1977. Relations entre la structure anatomique et l'amplitude du retrait du bois. Holzforschung, 31 (6), 194-200. 
Trendelenburg R., 1939. Das Holz als Rohstoff. 1st. ed. J.F. Lehmann Verlag, München.

Tsoumis G., 1958. [Growth, specific gravity and shrinkage of the wood of Pinus nigra Arn., Fagus silvatica L., Quercus sessiliflora Sm. and Castanea vesca Gärtn]. Aristotelian University, Thessaloniki.

Venet J., 1972. Utilisation et sylviculture du hêtre. Cours de l'Ecole Nationale du Génie Rural, des Eaux et des Forêts, Nancy.

WoвST H., 1967. Auswirkungen der Rotverkernung von Buchenstammholz auf einige kennzeichnende physikalische und mechanisch-technologische Eigenschaften. Proc. 14th Congr. I.U.F.R.O., Munich, pt. IX, Sect. 41, 179-209. 